\title{
Three-dimensional modeling of the neutron spectrum to infer plasma conditions in cryogenic inertial confinement fusion implosions
}

F. Weilacher, P. B. Radha, and C. Forrest

Citation: Physics of Plasmas 25, 042704 (2018); doi: 10.1063/1.5016856

View online: https://doi.org/10.1063/1.5016856

View Table of Contents: http://aip.scitation.org/toc/php/25/4

Published by the American Institute of Physics

\section{Articles you may be interested in}

A comprehensive alpha-heating model for inertial confinement fusion

Physics of Plasmas 25, 012703 (2018); 10.1063/1.4991405

In-flight neutron spectra as an ICF diagnostic for implosion asymmetries

Physics of Plasmas 25, 022705 (2018); 10.1063/1.5018108

Bulk hydrodynamic stability and turbulent saturation in compressing hot spots

Physics of Plasmas 25, 042703 (2018); 10.1063/1.5026413

Capsule physics comparison of National Ignition Facility implosion designs using plastic, high density carbon, and beryllium ablators

Physics of Plasmas 25, 032703 (2018); 10.1063/1.5016874

A near one-dimensional indirectly driven implosion at convergence ratio 30

Physics of Plasmas 25, 056311 (2018); 10.1063/1.5017976

High-energy-density plasma jet generated by laser-cone interaction

Physics of Plasmas 25, 042706 (2018); 10.1063/1.5021137

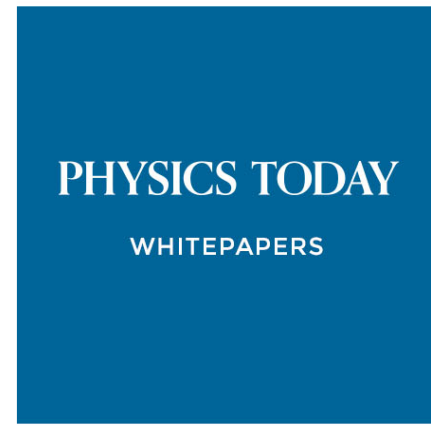

ADVANCES IN PRECISION MOTION CONTROL

Piezo Flexure Mechanisms and Air Bearings
READ NOW

PRESENTED BY

PI 


\title{
Three-dimensional modeling of the neutron spectrum to infer plasma conditions in cryogenic inertial confinement fusion implosions
}

\author{
F. Weilacher, ${ }^{\text {a) }}$ P. B. Radha, ${ }^{\text {b) }}$ and C. Forrest \\ Laboratory for Laser Energetics, University of Rochester, 250 East River Road, Rochester, \\ New York 14623-1299, USA
}

(Received 21 November 2017; accepted 26 March 2018; published online 26 April 2018)

Neutron-based diagnostics are typically used to infer compressed core conditions such as areal density and ion temperature in deuterium-tritium (D-T) inertial confinement fusion (ICF) implosions. Asymmetries in the observed neutron-related quantities are important to understanding failure modes in these implosions. Neutrons from fusion reactions and their subsequent interactions including elastic scattering and neutron-induced deuteron breakup reactions are tracked to create spectra. It is shown that background subtraction is important for inferring areal density from backscattered neutrons and is less important for the forward-scattered neutrons. A three-dimensional hydrodynamic simulation of a cryogenic implosion on the OMEGA Laser System [Boehly et al., Opt. Commun. 133, 495 (1997)] using the hydrodynamic code HYDRA [Marinak et al., Phys. Plasmas 8, 2275 (2001)] is post-processed using the tracking code IRIS3D. It is shown that different parts of the neutron spectrum from the view can be mapped into different regions of the implosion, enabling an inference of an areal-density map. It is also shown that the average areal-density and an areal-density map of the compressed target can be reconstructed with a finite number of detectors placed around the target chamber. Ion temperatures are inferred from the width of the D-D and D-T fusion neutron spectra. Backgrounds can significantly alter the inferred ion temperatures from the D-D reaction, whereas they insignificantly influence the inferred $\mathrm{D}-\mathrm{T}$ ion temperatures for the areal densities typical of OMEGA implosions. Asymmetries resulting in fluid flow in the core are shown to influence the absolute inferred ion temperatures from both reactions, although relative inferred values continue to reflect the underlying asymmetry pattern. The work presented here is part of the wide range of the first set of studies performed with IRIS3D. This code will continue to be used for post-processing detailed hydrodynamic simulations and interpreting observed neutron spectra in ICF implosions. Published by AIP Publishing.

https://doi.org/10.1063/1.5016856

\section{INTRODUCTION}

In direct-drive cryogenic inertial confinement fusion (ICF) ${ }^{1}$ implosions, a target comprising a shell of cryogenic deuterium-tritium (DT) fuel enclosing a vapor region is irradiated using multiple nearly identical laser beams. As the kinetic energy of the imploding shell is converted to the hotspot thermal energy, the shell decelerates, resulting in increased temperature and pressure required for fusion reactions to occur. Conditions achieved in the compressed core, at the time of peak fusion neutron production, of a typical cryogenic direct-drive implosion on the OMEGA Laser System $^{2}$ are shown in Fig. 1. The profile was obtained from a spherically symmetric simulation using the code LILAC; ${ }^{3}$ nonuniformity was ignored in this calculation. Ideally, a hot core is surrounded by a high-density shell, although two- or three-dimensional (2-D or 3-D) simulations indicate that while this is largely true, the neutron-producing region is typically not centered and the high-density shell can be significantly perturbed. Perturbations are typically quantified in terms of spherical harmonics (in 3-D simulations) or

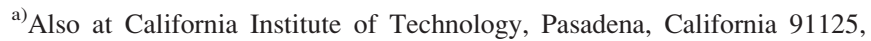
USA.

b) Author to whom correspondence should be addressed: rbah@lle.rochester.edu
}

Legendre polynomials (in 2-D experimental images or 2-D simulations).

The goal of cryogenic implosions on OMEGA is to understand the physics of directly driven ICF implosions. This requires observations that could potentially shed light on failure mechanisms including the role of long-wavelength asymmetries on implosion performance. Three-dimensional direct-drive simulations that include the effect of long-wavelength asymmetries performed with the arbitrary Lagrangian-Eulerian (ALE) code $H Y D R A^{4}$ indicate that significant long-wavelength asymmetries corresponding to spherical harmonics $\lambda=1,2$, up to 4 should be present during the time of neutron production. These asymmetries might be seeded by the power imbalances among the 60 beams of OMEGA or from nonuniformities on the inside of the cryogenic layer, at the ice/vapor interface, or by the initial error in the placement of the target relative to the center of the target chamber, etc. Long-wavelength asymmetries can compromise performance by reducing the volume over which neutrons are produced. In Fig. 1, the volume over which neutrons are produced has a radius of $\sim 20 \mu \mathrm{m}$. The Rayleigh-Taylor ${ }^{5}$ growth of the nonuniformities during the deceleration phase of the imploding capsule results in growth at approximately this radius; spikes of the high-density shell penetrate the hot spot, whereas bubbles of lower-density 


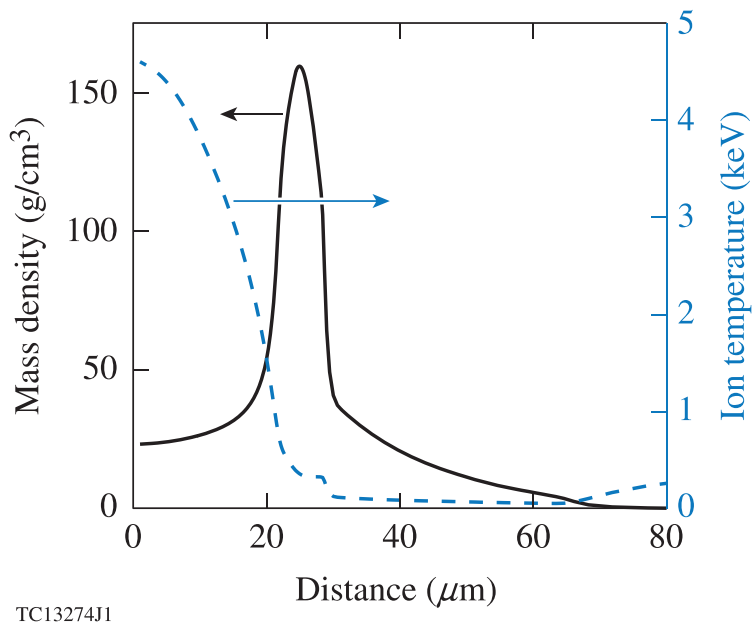

FIG. 1. Typical density and temperature profile at peak neutron production in an OMEGA cryogenic implosion simulated using the spherically symmetric code $L I L A C^{3}$ (see the text for details).

material distort the high-density shell. These large bubbles in the high-density shell may permit heat and fuel to escape, thereby decreasing fusion yields. These asymmetries result in angular variations in areal density [defined as $\rho R$ $=\int_{0}^{R} \rho(r) \mathrm{d} r$, the radial integral of the areal density, where $\rho(r)$ is the density along the radius of the target and $R$ is the outer radius of the target] around the compressed core. Another manifestation of these asymmetries is the large-scale fluid flow resulting in residual kinetic energy, i.e., kinetic energy that has not been converted to hot-spot energy. Diagnosing these asymmetries is therefore important for identifying a potential source of performance degradation. Until recently, x-ray images from a single view have been used to infer the existence of asymmetries in OMEGA cryogenic implosions, ${ }^{6,7}$ although quantitative measures are outstanding. Multiple views of neutron-based diagnostics have been available, although a tool to interpret the combined results has been unavailable.

In this work, we show that with additional views of the neutron-based diagnostics, asymmetries can potentially be diagnosed on OMEGA. Results from the newly developed code IRIS3D (see the Appendix), for post-processing 3-D hydrodynamic simulations, are described here. First, the impact of various types of backgrounds on different parts of the neutron spectra used to determine areal density and its asymmetries is discussed. Second, the use of a single view or multiple views to infer areal-density asymmetries is elaborated upon. Third, the detection of asymmetries through the effect of the residual fluid flow on neutron spectrum is described. This work is the first of such an analysis for direct-drive cryogenic OMEGA implosions and comprises tests of the IRIS3D code; future work will include detailed post-processing of 3-D simulations to compare quantities derived from neutron spectra with experiment.

The generalized Lawson criterion for ICF implosions ${ }^{8}$ provides a measure of implosion performance and can be written for OMEGA scale as

$$
\chi_{1-\mathrm{D}} \approx\langle\rho R\rangle\left(\mathrm{g} / \mathrm{cm}^{2}\right)\left[\frac{\langle T\rangle(\mathrm{keV})}{4.4}\right]^{0.8}
$$

where $\langle\rho R\rangle$ is the neutron-weighted areal density of the compressed target and $\langle T\rangle$ is the neutron-weighted ion temperature in the hot spot. A value of $\chi_{1-\mathrm{D}} \sim 1$ indicates marginal ignition, where the ratio of the output fusion neutron energy to the input laser energy is $\sim 1$. This form of the generalized Lawson criterion is derived from a power law fit to a series of spherically symmetric simulations and is written in terms of quantities - neutron-weighted areal density and ion temperature-that are, in principle, observable. As discussed later, the areal density is inferred from the elastically scattered neutron spectrum ${ }^{9-11}$ and ion temperature is inferred from the width of the neutron spectrum of the $\mathrm{D}-\mathrm{T}$ fusion neutrons. ${ }^{12}$ Asymmetrically driven implosion experiments limit, however, the ability to directly compare the experimentally inferred quantities with spherically symmetric simulations. $\langle\rho R\rangle$ can vary spatially in an implosion and the inferred value depends on the viewing direction. Similarly, fluid flow in the hot spot can change the width of the neutron spectrum resulting in direction-dependent inferences of apparent ion temperature. Therefore, multiple measurements are required to constrain the values attained in implosion experiments. In addition, certain neutron-producing reactions in an implosion such as the deuteron breakup reaction or $\mathrm{T}-\mathrm{T}$ fusion reactions can introduce ambiguities in the interpretation of the neutron spectrum. Therefore, it is important to understand the role of backgrounds and perform studies on different patterns of asymmetry to be able to interpret observations. Simultaneously, comparing results from 3-D hydrodynamic simulations to observations is necessary to identify the adequacy of the modeling and infer the role of nonuniformity seeds in experiments. This requires a new analysis tool to post-process 3-D simulations and permit comparison of observables with experiment.

In this paper, a Monte Carlo neutron-tracking code IRIS3D (see the Appendix) is used to model neutron transport from three primary fusion reactions in a DT capsule: the 14.1-MeV D-T fusion neutrons, the 2.45-MeV D-D fusion neutrons, and the $\mathrm{T}-\mathrm{T}$ three-body reaction, which results in a continuum of neutrons. Additionally, three secondary interactions of the primary D-D and D-T neutrons including their elastic scattering off the deuterium and tritium ions (used to diagnose areal density) and the neutron-induced deuteron breakup reaction (which is a background to the elastically scattering reactions) are also modeled. Neutron-induced triton breakup is not included in this work because its cross section is $\sim 4 \times$ smaller than the deuteron breakup reaction. ${ }^{13}$ In Sec. II, we describe the basic well-known relationship between $\rho R$ and elastic scattering. ${ }^{9-11,14}$ In Sec. III, we indicate the effect of neutrons from other fusion processes in the compressed target on the inference of $\langle\rho R\rangle$, i.e., the role of backgrounds. In Sec. IV, we exploit the kinematics of the elastic scattering reaction to infer the spatial dependence of the areal density in an implosion from one view. As far as we know, the concept presented in this work to map parts of the measured neutron spectrum to different regions of the implosion is new. The inference of areal-density asymmetries with multiple spectrometers is also discussed. Inferring ion temperatures from neutron spectral widths is discussed in 
Sec. V. It is shown that fluid flows increase the neutron spectral widths, leading to an increase in the apparent inferred ion temperature. Therefore, significantly different values of apparent ion temperature determined in different directions around an implosion should indicate the presence of asymmetries. While this effect has been modeled previously, ${ }^{15}$ the work presented here is a demonstration of the capabilities of IRIS3D. It is also shown that while the absolute values of the inferred temperatures might be inaccurate, the relative values still potentially track the underlying asymmetry. Finally, conclusions are presented in Sec. VI.

\section{AREAL DENSITY AND ELASTIC SCATTERING}

The number of neutrons that scatter elastically can be expressed as

$$
y_{\mathrm{n}}^{\prime}=\int_{s} y_{\mathrm{n}} n \sigma \mathrm{d} s,
$$

where $y_{\mathrm{n}}$ is the number of primary neutrons, $s$ is the distance along the path of the primary neutron, $n$ is the number density of deuterons or tritons (particles per unit volume), and $\sigma$ is the cross section of the scattering interaction. For the D-T primary neutrons at $14.1 \mathrm{MeV}$, the cross section for scattering off deuterons, $\sigma=\sigma_{\mathrm{d}} \approx 640 \mathrm{mb}$, and for scattering off the background tritons, $\sigma=\sigma_{\mathrm{t}} \approx 930 \mathrm{mb}$. If the substitution

$$
n=\frac{\rho}{\bar{m}} f
$$

is used, where $\bar{m}$ represents the average mass of particles in the material and $f$ represents the fraction of particles that are of the type of interest (for DT fuel with equal proportions of $\mathrm{D}$ and $\mathrm{T}, M=2.5 \mathrm{amu}$ and $f=1 / 2$ ), Eq. (3) can be rewritten as

$$
y_{\mathrm{n}}^{\prime}=\int_{s} y_{\mathrm{n}} \frac{\rho}{\bar{m}} f \sigma \mathrm{d} s .
$$

The number of primary neutrons, $y_{\mathrm{n}}$, is not constant along the path length since a fraction undergoes various interactions in the compressed target. However, $y_{\mathrm{n}}$ typically changes by less than $10 \%$ along a path. For the purpose of illustrating the dependencies, it is assumed to be constant. The code used in this paper takes into account the reduction of $y_{\mathrm{n}}$ along a path. Therefore, since $\sigma$ and $f$ are constants along the path length of the neutrons, the ratio of the scattered to primary neutrons can be written as (also called the down-scatter ratio or DSR)

$$
\frac{y_{\mathrm{n}}^{\prime}}{y_{\mathrm{n}}} \approx \sigma f \int_{s} \rho(s) \mathrm{d} s .
$$

Including scattering off both the deuterons and tritons, one can write an expression for the total DSR as

$$
\mathrm{DSR} \equiv \frac{y_{\mathrm{n}}^{\prime}}{y_{\mathrm{n}}} \approx \frac{f_{D} \sigma_{\mathrm{D}}+f_{T} \sigma_{T}}{\bar{m}} \cdot \rho L,
$$

where $\rho L=\int_{0}^{L} \rho \mathrm{d} s$ is the areal density along a total path length $L$. The DSR is the observed quantity in the experiment used to infer areal density. If the trajectories of the neutrons were purely radial, $\rho L$ would correspond to $\rho R$, where $R$ is the radius of the plasma. Since fusion reactions occur throughout the hot core, neutron trajectories are not radial, even for spherically symmetric implosions. Therefore, comparisons between the calculated and the observed DSR are required to identify if the simulated areal density has been achieved in implosion experiments. In addition, $\rho L$ is dominantly sampled when the neutron-production rate is the highest in the experiment. Consequently, the areal density inferred in the experiment corresponds to a neutronweighted value, or $\langle\rho L\rangle$. Therefore, the inference of an areal density from a neutron spectrum results in a value for $\langle\rho L\rangle$, whereas lineouts from the center of a simulated profile weighted by neutron production would provide a measure for $\langle\rho R\rangle$.

To test and illustrate the physics associated with neutron interaction and transport, an "ice-block" profile (Fig. 2) that approximates typical profiles in a cryogenic implosion is used. This "ice-block" model is characterized by regions of constant density and temperature. Using a Monte Carlo approach, neutrons are transported through this profile and the spectrum of the emitted neutrons is calculated (Fig. 3). Calculated cross sections for elastic scattering, ${ }^{16}$ which previously have shown excellent agreement with measurements, ${ }^{16}$ were used in this calculation. This Monte Carlo code, which post-processes only spherically symmetric simulations, has also been compared previously with experiment and shown to be in excellent agreement when detailed capsule simulations are post-processed to obtain spectra for a range of implosions. ${ }^{17}$ Of note in the neutron spectrum are the DT primary peak at $14.1 \mathrm{MeV}$ and the deuteron and triton backscattered edges at 1.5 and $3.5 \mathrm{MeV}$, respectively.

The DSR is inferred from different parts of the neutron spectrum on OMEGA. Two methods are used: (1) the magnetic recoil spectrometer (MRS) ${ }^{10}$ is used to measure the down-scattered neutron spectrum; the DSR between 10 and $12 \mathrm{MeV}$ is used to infer $\langle\rho L\rangle$. Neutrons incident on a deuterated plastic (CD) foil are elastically scattered to produce

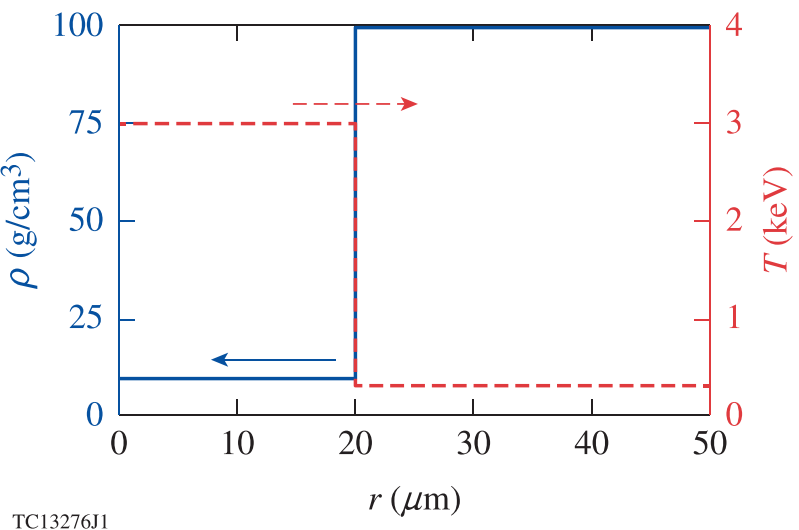

FIG. 2. The spherically symmetric "ice-block" used to describe typical density and temperature profiles in a cryogenic DT implosion on OMEGA. A $50-50$ DT fuel ratio is used. 


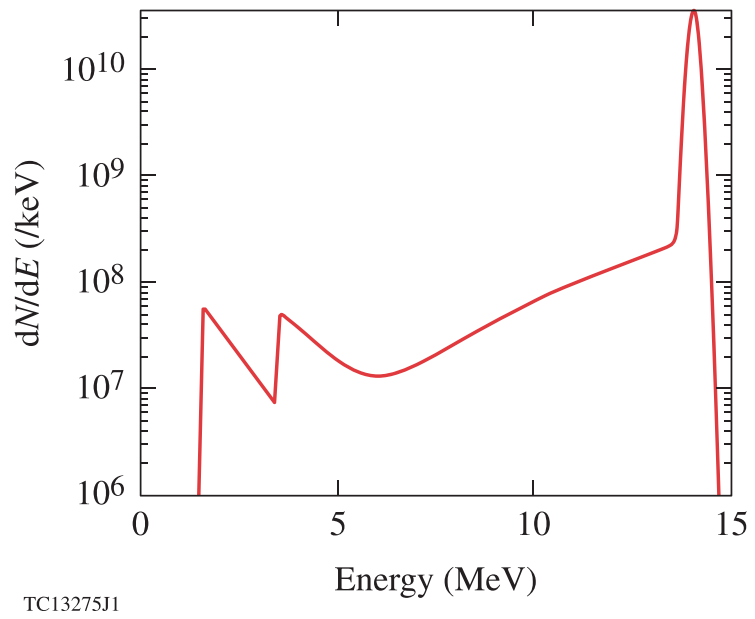

FIG. 3. Example of a simulated neutron spectrum including only D-T primary neutrons elastically scattered off deuterons and tritons.

deuterons, which are then dispersed in energy using a magnet and counted using a plastic (CR-39) foil. (2) Neutron time of flight (nTOF) is used to measure the backscattered neutron spectrum: ${ }^{11}$ the ratio between 3.5 and $4.5 \mathrm{MeV}$ provides a measure of $\langle\rho L\rangle$ although energies up to $6 \mathrm{MeV}$ are measured currently. Extension to higher energy ranges is possible. Activation techniques ${ }^{18}$ mounted at different lines of sight can, in principle, provide direct information of the $\langle\rho L\rangle$ variation. The variation in neutron yields among the fixed nuclear activation diagnostics is proportional to the variation in $\langle\rho L\rangle$. For the typical areal densities in OMEGA cryogenic implosions, $\sim 200 \mathrm{mg} / \mathrm{cm}^{2}$, only $\sim 3 \%$ of neutrons are scattered via neutron interactions, making them less useful for OMEGA.

Kinematically, DSR inferred from a specific energy range and from a particular direction samples $\langle\rho L\rangle$ from only a specific portion of the implosion. If a neutron (or any particle) of mass $m$ and with an initial energy $E$ scatters elastically off of a particle with mass $M$ and emerges with an energy $E^{\prime}$, its angle of scattering $\theta$ is given by

$$
\cos \theta=\frac{x(A+1)-(A-1)}{2 \sqrt{x}}
$$

where $A=M / m$ and $x=E^{\prime} / E$.

As a result, neutrons deflected by small angles lose a small amount of energy, whereas neutrons that exhibit nearly backscattered angles lose a significant amount of energy. As a consequence, different parts of the neutron spectrum measured from a single spectrometer can be mapped to different parts of the implosion. The open squares in Fig. 4 show the locations of the MRS and nTOF detectors in the OMEGA target chamber. The contours represent $1-\mathrm{MeV}$ energy ranges over the regions in the detector's view under the assumption of a point neutron source from the center for scattering off deuterons [Figs. 4(a) and 4(c)] and tritons [Figs. 4(b) and 4(d)]. An extended source would spatially blur the regions but not significantly enough to change the conclusions. The $10-$ to $12-\mathrm{MeV}$ range viewed by the MRS has the advantage of focusing in on a narrower region of the target; however, a large number of detectors viewing this (a)

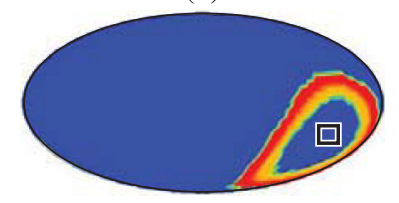

(c)

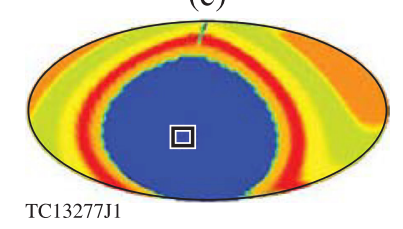

(b)

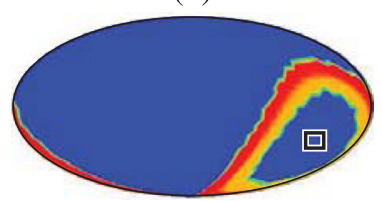

(d)

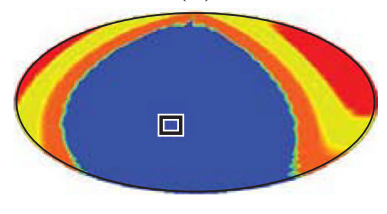

FIG. 4. (a) Region probed by the magnetic recoil spectrometer (MRS) detector when measuring elastically scattered neutrons odd deuterium ions in the $10-$ to $12-\mathrm{MeV}$ range. The location of the detector in the OMEGA target chamber is shown as a black square. (b) Same as (a) but for neutrons elastically scattering off tritons. [(c), (d)] Same as (a) and (b) but for a time-offlight detector measuring backscattered neutrons in the 1 - to $6-\mathrm{MeV}$ range. The black square in each figure indicates the locations of the spectrometers in the OMEGA chamber. The contours are given in 1-MeV steps.

range would be necessary to get full, $4 \pi$ sampling coverage as a result. Multiple MRS-type spectrometers for the higher energy range can be expensive and occupy significant real estate in the OMEGA target chamber. Therefore, multiple views at lower energies (i.e., through time-of-flight measurements), which can map broader regions of the target, are considered in this paper.

For an nTOF detector, using a range of 1 to $6 \mathrm{MeV}$, a much larger portion of the target can be sampled [Figs. 4(c) and 4(d)]. Note that only the higher three sub-rings are visible for triton backscatter [Fig. 4(d)] since the backscattered peaks for triton scattering occur around $3.5 \mathrm{MeV}$. Breaking up this spectrum into smaller sub-ranges makes it possible for a spectrometer to sample $\langle\rho L\rangle$ with a higher spatial resolution. In addition, by using multiple spectrometers, not only $\langle\rho L\rangle$ but also asymmetry in $\langle\rho L\rangle$ can be mapped. As discussed in Sec. III, however, backgrounds can significantly influence the spectrum at these lower energies and must be accounted for carefully.

\section{BACKGROUND ISSUES AFFECTING THE DETERMINATION OF $\langle\rho L\rangle$}

The spectrum shown in Fig. 3 includes only the primary and the elastically scattered neutrons. Additional effects such the broadening of the neutron spectrum resulting from thermal effects, bulk fluid motion, and multiple scattering are discussed in this section.

Multiple scattering is taken into account in the particle tracking code by recursively scattering neutrons off the fuel ions until the effect is no longer numerically significant. Figure 5 demonstrates the effect that multiple scattering can have on down-scattered spectra for two different areal densities. Because DSR is approximately proportional to $\langle\rho L\rangle$, the number of multiply scattered neutrons should be proportional to some power of $\langle\rho L\rangle$. Consequently, the effect of multiple scattering should become more significant as $\langle\rho L\rangle$ increases. This is shown in Fig. 5, where for two ice-block profiles with different areal densities, the neutron spectrum is calculated 


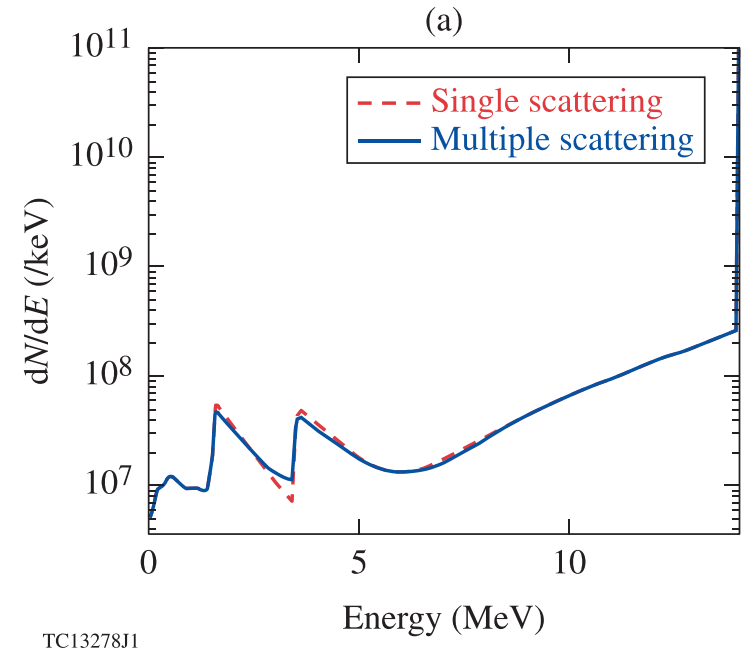

(b)

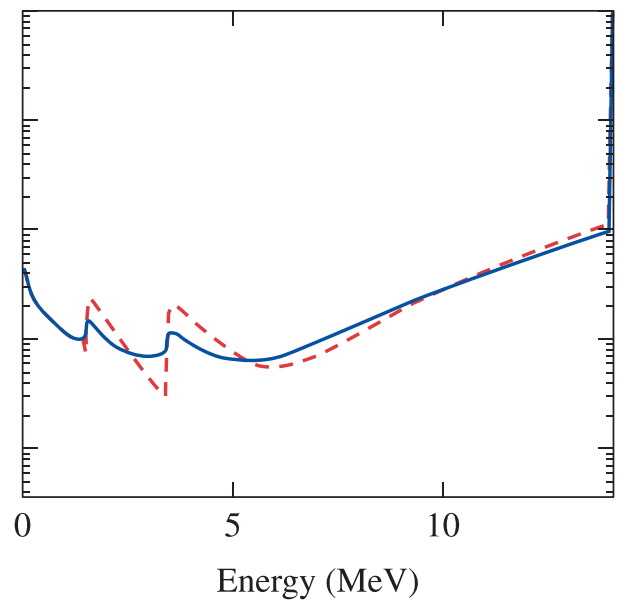

FIG. 5. Neutron spectrum generated by IRIS3D considering only D-T primary neutrons and neutrons generated from elastic scattering, with (solid) and without multiple (dashed) scattering: (a) for the ice-block profile shown in Fig. 3, which has a $\rho R$ of $0.32 \mathrm{~g} / \mathrm{cm}^{2}$; and (b) for the same profile, but with a higher shell density of $500 \mathrm{~g} / \mathrm{cm}^{3}$, resulting in a $\rho R$ of $1.52 \mathrm{~g} / \mathrm{cm}^{2}$. In the case of the higher $\rho R$, it is clear that multiple scattering has a larger impact on the spectrum, particularly in the backscattered region (1 to $6 \mathrm{MeV})$.

with and without multiple scattering. For the higher areal density, characteristic of cryogenic implosions on the NIF, significant differences are observed in the neutron spectra at lower energies, indicating that multiple scattering is important under such conditions. For OMEGA-scale implosions, areal densities are typically around 0.15 to $0.25 \mathrm{~g} / \mathrm{cm}^{2}$. In this situation, the effect of multiple scattering is negligible and can be ignored in the calculations.

All significant components of the neutron spectrum from a cryogenic DT implosion are shown in Fig. 6. Again, the iceblock model in Fig. 2 is used to calculate the spectrum. Two additional primary neutron-generating reactions are likely to occur in a DT-filled capsule: $d(d, n)^{3} \mathrm{He}$ and $t(t, 2 n)^{4} \mathrm{He}$. DD primary neutrons, generated around $2.45 \mathrm{MeV}$, are not a significant background to the down-scattered neutrons since they are

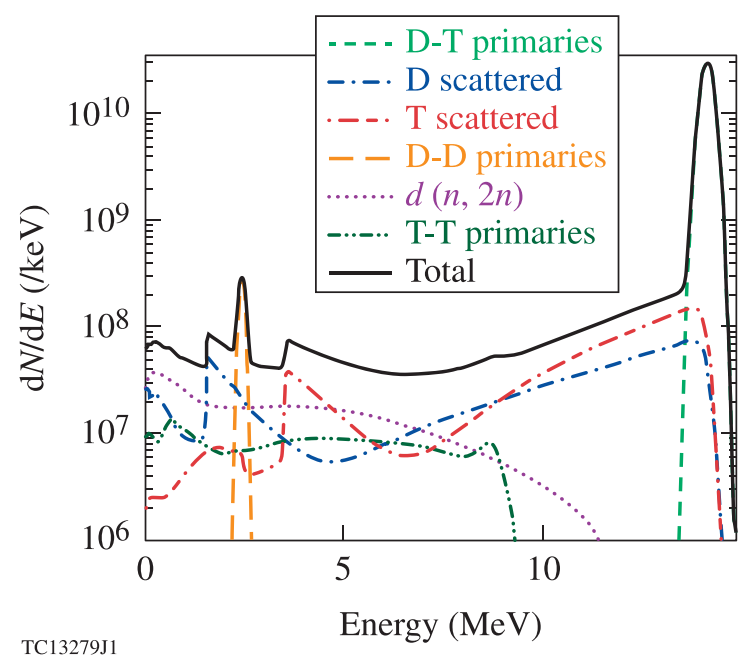

FIG. 6. Neutron spectra generated by IRIS3D including all relevant reaction channels. Broadening from thermal effects, bulk fluid flow, and multiple scattering are included in the calculation. The width of the D-T neutron peak (14.1 MeV) is a function of ion temperature and bulk fluid flow in the hot core. Down scattered neutrons in the 10 to $12-\mathrm{MeV}$ range (used for $\langle\rho L\rangle$ measurements) are unaffected by other background sources such as neutrons from the deuteron breakup reaction and $\mathrm{T}-\mathrm{T}$ fusion reactions. clearly recognizable in the spectrum. They can interact, however, with the cold fuel through, for example, elastic scattering, causing them to contribute to the background below $2.45 \mathrm{MeV}$ - for example, around the deuteron backscattered peak.

Primary neutrons from the $\mathrm{T}-\mathrm{T}$ reaction, on the other hand, do affect the neutron measurement in the backscattered region. Because the $\mathrm{T}-\mathrm{T}$ reaction produces three products, the energies of the outgoing neutrons can be anywhere from 0 up to almost $10 \mathrm{MeV}$. This causes them to act as a background to the scattered neutron spectrum. Unlike the number of backscattered or down-scattered neutrons, the number of neutrons from the $\mathrm{T}-\mathrm{T}$ reactions is independent of $\langle\rho L\rangle$. To the best of our knowledge, this reaction is isotropic and this background is assumed to be independent of the viewing angle.

Neutrons produced from the deuteron breakup reaction, $\mathrm{d}(\mathrm{n}, 2 \mathrm{n}) \mathrm{p}$, are yet another source of background to the backscattered neutron spectrum. Similar to the elastic-scattering reaction, this reaction is proportional to $\langle\rho L\rangle$. In IRIS3D, this reaction is calculated only for DT primary neutrons because of the unavailability of cross sections for other energies. With an end point of $11.8 \mathrm{MeV}$, the spectrum of neutrons from the deuteron breakup reaction mainly constitutes background in the lower-energy portion of the spectrum. Note that when areal-density asymmetries are present, this reaction can result in ambiguities in the interpretation of areal densities from the down-scattered neutron spectrum. The directionality of neutrons from this reaction is shown in Fig. 7. Neutrons are launched radially from the center of the target and the emitted neutrons are plotted in neutrons per steradian as a function of angle. Figure 7 shows that the large majority of deuteron-breakup neutrons emerge in the forward direction, but because there is no 1-to-1 correspondence between energy and scattering angle for an individual deuteron-breakup neutron (because there are three product particles), even these forward-emerging neutrons can have arbitrarily low outgoing energies. Therefore, an exact 


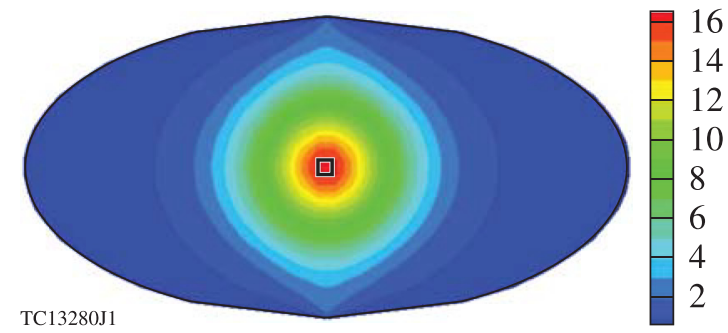

FIG. 7. Projection of the angular distribution of the deuteron breakup reaction with respect to a synthetic detector located at the center (black square).

interpretation of the down-scattered neutron spectrum is challenging since it requires knowledge of the areal-density asymmetry to subtract the background components.

The cumulative neutron spectrum (including all backgrounds) is shown in Fig. 8. The figure indicates that at lower energies, backgrounds can significantly influence the spectrum and therefore the inferred areal density. This is also summarized in Table I, which indicates that the $\langle\rho L\rangle$ inferred from the observed spectra in the 3.5- to 4.5-MeV range when one does not account for backgrounds is considerably larger than the areal density, whereas backgrounds have a marginal effect on the inferred areal density in the 10 - to $12-\mathrm{MeV}$ range.

\section{DIAGNOSING $\langle\rho L\rangle$ ASYMMETRIES}

Backgrounds can also have an effect on the observation of $\langle\rho L\rangle$ asymmetries. Figure 9 shows how the detection of areal density around an asymmetric target can be affected by the inclusion of backgrounds for two different asymmetry patterns. Asymmetric profiles are obtained by perturbing the ice-block profiles with Legendre modes. To illustrate the effect, a $\lambda=1$ pattern with a peak-to-valley amplitude of $0.2 \mathrm{~g} / \mathrm{cm}^{2}$ is imposed on the profile [Fig. 9(a)]. In what follows, it is assumed that infinite coverage around the target chamber is available. When the $3.5-$ to $4.5-\mathrm{MeV}$ range is used to infer areal density, the opposite phase is measured

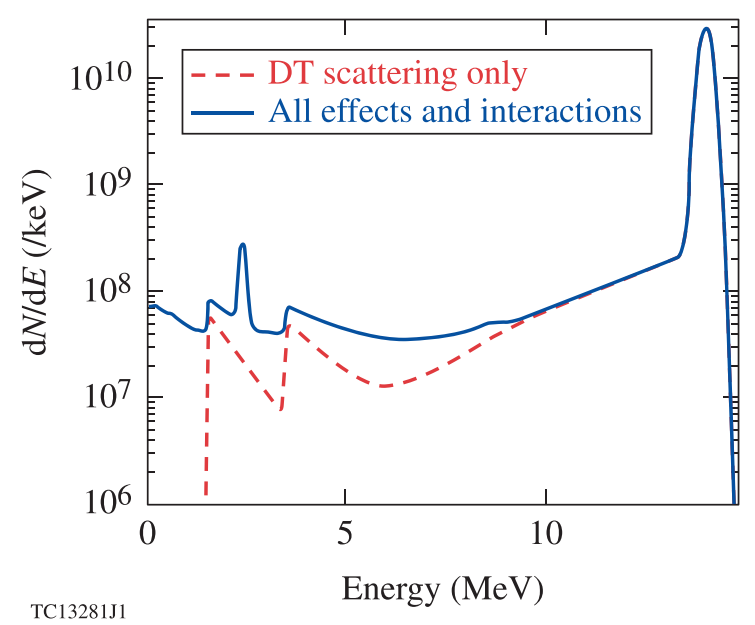

FIG. 8. Spectra generated by IRIS3D for the profile shown in Fig. 2 with (solid) and without (dashed) the inclusion of neutrons background reactions. The $\langle\rho L\rangle$ inferred from each spectrum in the energy ranges of 3.5 to 4.5 MeV and 10 to $12 \mathrm{MeV}$ is shown in Table I.
TABLE I. The $\rho L$ values inferred from the spectra shown in Fig. 8 over thespecified energy ranges. These values should be compared to a target $\rho R$ $\sim 0.32 \mathrm{~g} / \mathrm{cm}^{2}$.

\begin{tabular}{lcc}
\hline \hline Inferred $\rho L\left(\mathrm{~g} / \mathrm{cm}^{2}\right)$ & 3.5 to $4.5 \mathrm{MeV}$ & 10 to $12 \mathrm{MeV}$ \\
\hline DT scattering only & 0.34 & 0.34 \\
All effects and interactions & 0.56 & 0.34 \\
\hline \hline
\end{tabular}

for the mode [Fig. 9(b)]. This is because the neutrons being observed are backscattered and originate from the opposite side of the target from the detector. The minimum and maximum observed areal densities when backgrounds are ignored are 0.27 and $0.41 \mathrm{~g} / \mathrm{cm}^{2}$, respectively, so the maximumobservable contrast is $\sim 0.14 \mathrm{~g} / \mathrm{cm}^{2}$-less than the imposed amplitude primarily because of the averaging of the target caused by the finite solid angle of the detectors. When background effects are considered, the $\lambda=1$ pattern is still clearly visible, but the maximum-observable contrast drops to $\sim 0.06 \mathrm{~g} / \mathrm{cm}^{2}$ [Fig. 9(c)]. Consequently, while absolute values are not inferred accurately, the overall asymmetry pattern is still encoded in the neutron spectrum if the pattern is dominated by $\lambda=1$.

Somewhat different results are obtained when a $\lambda=2$ mode is imposed on the profile [Fig. 9(a)]. The $\lambda=2$ pattern is also apparent whether or not background effects are considered. Additionally, the phase of the underlying arealdensity pattern is reproduced by the inferred areal density [Fig. 9(b)]. Since the applied asymmetry is an even mode, sampling the rear of the implosion actually results in the same $\langle\rho L\rangle$ being sampled as would have been seen when sampling the front of the implosion.

The maximum-observable contrast drops only marginally when all backgrounds are considered; $\sim 0.075 \mathrm{~g} / \mathrm{cm}^{2}$ with all background sources compared to $\sim 0.080 \mathrm{~g} / \mathrm{cm}^{2}$. As a result, the inclusion of backgrounds does not prevent the diagnosis of the $\lambda=2$ asymmetry. This is caused by the opposite parities of the $\lambda=1$ and $\lambda=2$ modes. Recall from Sec. III that (1) the deuteron-breakup background is $\langle\rho R\rangle$ dependent, (2) its neutrons emerge mostly in the forward direction (see Fig. 7), and (3) it is most significant in the backscattered (low-energy) portion of the neutron spectrum, including the $3.5-$ to $4.5-\mathrm{MeV}$ range. Therefore, when diagnosing $\langle\rho L\rangle$ using neutron energies in this energy range, $\langle\rho L\rangle$ is sampled simultaneously from the opposite side of the target via elastically backscattered neutrons and the front side of the target via deuteron-breakup neutrons. In oddmode asymmetries, these two locations will always have opposite areal densities, hindering the detection of those asymmetries, while for even-mode asymmetries, they will always have equal areal densities, enhancing the detection of the asymmetries. Also note that the contrast is reduced relative to the $0.2 \mathrm{~g} / \mathrm{cm}^{2}$ amplitude of the imposed mode. This is caused by the shorter wavelength of the asymmetry and the finite solid angle of the detector.

The decreasing contrast with increasing mode number is demonstrated in Fig. 10. Even with infinite coverage and backgrounds ignored, the contrast for an imposed mode with $\lambda=4$ falls to $8 \%$ of the imposed value for the backscattered 
(a)

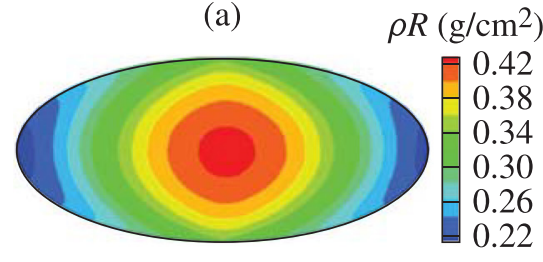

(d)

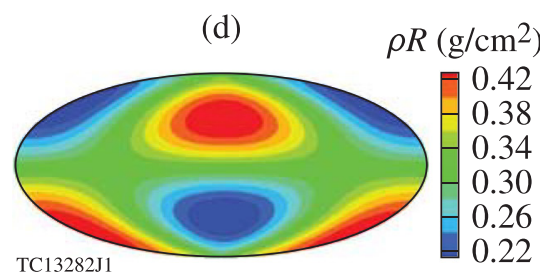

(b)

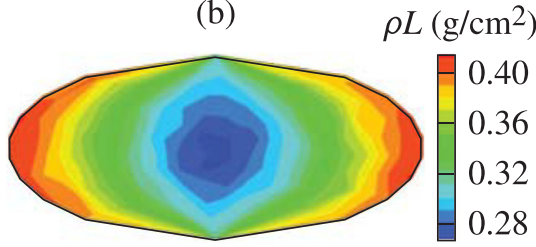

(e)

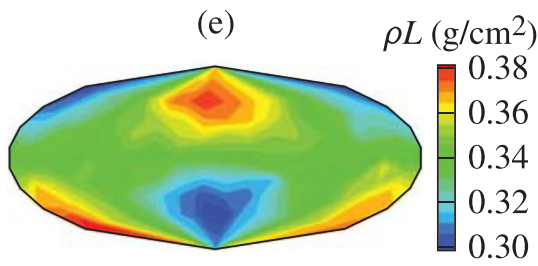

(c)

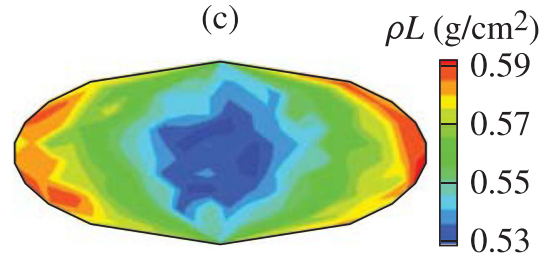

(f)

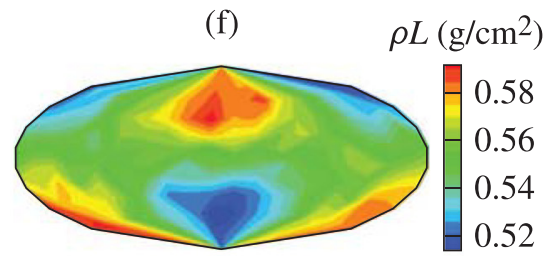

FIG. 9. (a) $\rho R$ map (Hammer plot) for a density profile with an imposed $\lambda=1$ asymmetry. The density and temperature profiles are the same as the ones shown in Fig. 2 but with a spherical harmonic perturbation applied to the density in each cell based on the cell location. (b) $\rho L$ map inferred from backscattered neutrons in the energy range of 3.5 to $4.5 \mathrm{MeV}$, which should be contrasted with the $\rho R$ map shown in (a). This map was generated by $I R I S 3 D$ when considering only D-T primaries and their single scattering off deuterons and tritons. (c) Same as (b), but with all reaction channels included in the calculation. [(d)-(f)] Same as (a)-(c), but an $\lambda=2$ mode imposed on the ice-block density profile.

neutrons (3.5 to $4.5 \mathrm{MeV}$ ) and to $17 \%$ for the forwardscattered neutrons (10 to $12 \mathrm{MeV}$ )—extremely low values that likely cannot be detected. This indicates that the limit in diagnosing asymmetries is likely longer than the wavelengths corresponding to $\lambda=4$. Note that this type of study is being used to test the code. It is not expected that detailed mode information can be obtained by measuring the scattered neutron yields from several different locations.

Background subtraction is the subject of active investigation for low-energy neutron spectra. In independent experiments, the neutron spectrum from the deuteron breakup reaction is being measured to infer the cross section of this interaction. This discussion is beyond the scope of this work. For the remainder of the paper, it is assumed that backgrounds can be accounted for.

An $\langle\rho L\rangle$ map of the implosion can be reconstructed as follows: For each spectrometer and specified energy range, a $\langle\rho L\rangle$ can be inferred from the observed yield in that energy range relative to the primary yield [Eq. (6)]. This inferred $\langle\rho L\rangle$ can be projected as a ring on a sphere enclosing the implosion as shown in Fig. 4. To illustrate this method, the deuteron and triton elastic scattering are averaged using an average value of $A=2.5$ when calculating projection angles. Applying this method for each spectrometer and energy range, rings that overlap can be averaged, resulting in a reconstructed $\langle\rho L\rangle$ map. Note that this projection assumes that neutrons are produced as a central source that introduces an error in the inferred $\langle\rho L\rangle$ maps. This error is potentially quantifiable with neutron images that indicate the location of the primary source in the compressed core. Neutron imaging has little use on OMEGA, however, because of limited neutron statistics in OMEGA cryogenic implosions; although, as shown below, if the perturbation were of a significantly large wavelength, the inference of areal-density variations would remain robust.

A proof-of-principle test of the reconstruction technique for $\langle\rho L\rangle$ was performed by post-processing 3-D HYDRA simulation of an OMEGA cryogenic implosion. The simulation includes realistic nonuniformity seeds from laser beam imbalances and the offset of the target in the target chamber. Primary D-T neutrons and elastically scattered neutrons were tracked for 20 time slices around peak neutron production. The density profile at peak neutron production shows a dominant $\lambda=1$ (Fig. 11) in the compressed core, primarily because the target was not positioned at the target chamber center The lineout of the areal density taken from the location of the peak neutron production is also shown in Fig. 11(b). With infinite coverage, the inferred $\langle\rho L\rangle$ map from the neutron spectrum is shown in Fig. 12(a). Since the dominant mode is given by $\lambda=1$, the phase is reversed compared to Fig. 11(b). Four detectors (a realistic number with future upgrades on OMEGA) were arranged in an ad hoc tetrahedral fashion around the target in IRIS3D, and the code was used to simulate their observed spectra from 1 to $6 \mathrm{MeV}$. The locations of these detectors are shown in Fig. 12(b). Neutron spectra are used to reconstruct an areal-density map using the procedure described previously. The shape of the reconstructed map compares well to the lineout map seen in Fig. 11(b), although the contrast is significantly lower, at about $0.07 \mathrm{~g} / \mathrm{cm}^{2}$, compared to about $0.26 \mathrm{~g} / \mathrm{cm}^{2}$ in the
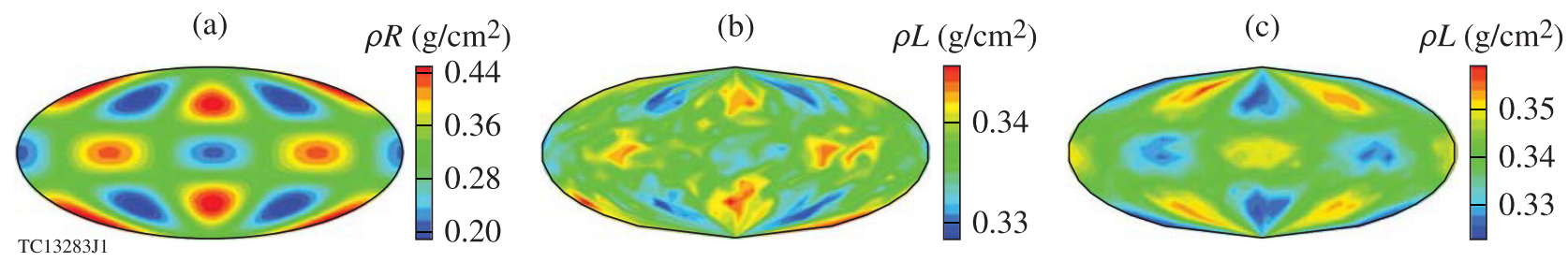

FIG. 10. (a) Same as Fig. 9(a), with an $\lambda=4$ asymmetry imposed on the density profile. (b) $\rho L$ map inferred from backscattered neutrons in the energy range of 3.5 to $4.5 \mathrm{MeV}$, which should be contrasted with the $\rho R$ map shown in (a). (c) Same as (b), but using the 10- to $12-\mathrm{MeV}$ energy range. 
(a)
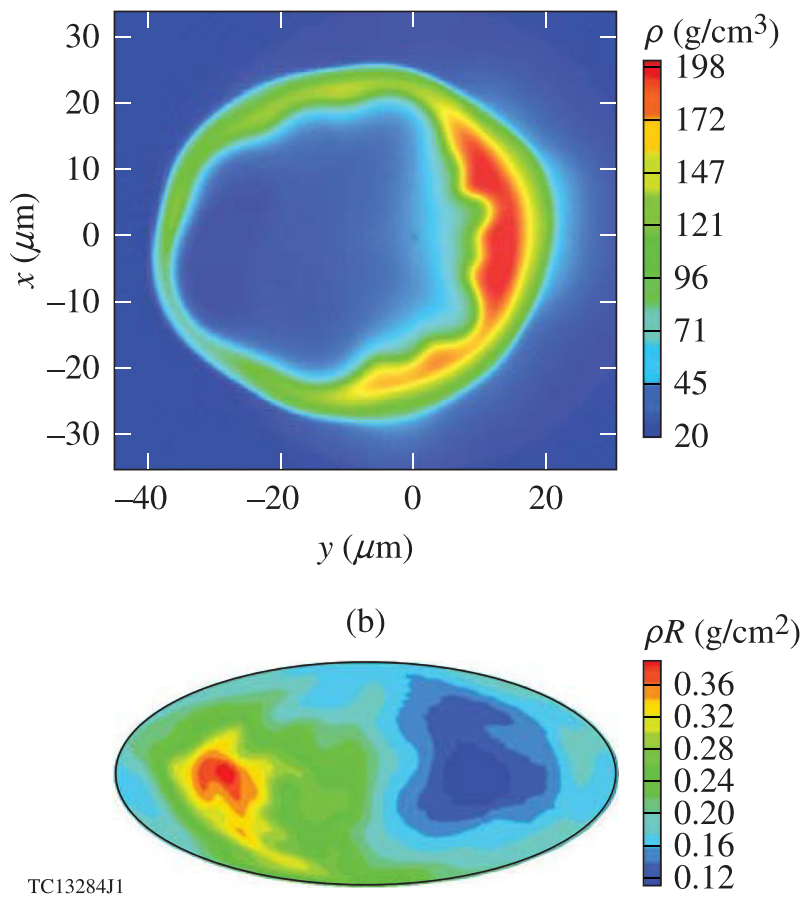

FIG. 11. (a) Equatorial cross section of the density profile generated by HYDRA. A $\lambda=1$ mode is apparent. (b) $\rho R$ map corresponding to the profile shown in (a). The $\lambda=1$ mode is again the dominant feature.

lineout or $\sim 27 \%$. Figure 12(a) suggests, however, that even with arbitrarily many detectors viewing the low-energy portion of the spectrum, the maximum-observable contrast is $\sim 0.10 \mathrm{~g} / \mathrm{cm}^{2}$. This suggests that if one can account for background, a small number of detectors have the potential to reconstruct accurate areal-density maps of low-mode asymmetries around a target. It should be noted, however, that the

(a)

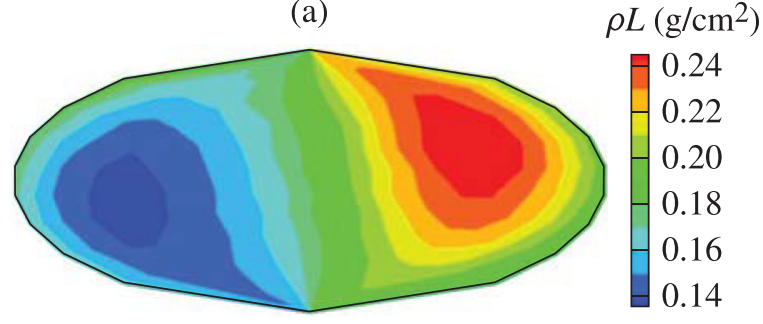

(b)

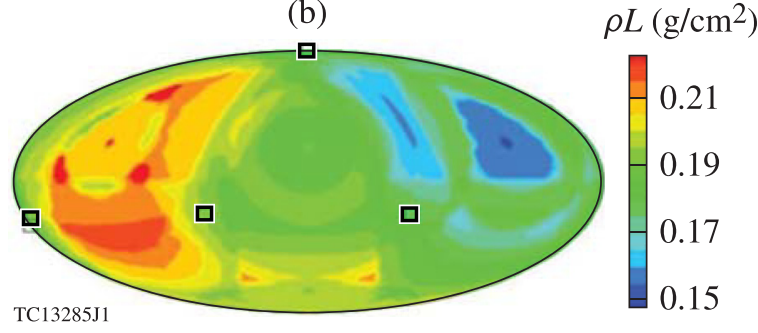

FIG. 12. (a) $\langle\rho R\rangle$ map (Hammer plot) associated with the profile shown in Fig. 11 when backscattered neutrons in the energy range of 3.5 to $4.5 \mathrm{MeV}$ are used. Note the expected phase change compared to Fig. 11(b). (b) Reconstructed $\langle\rho L\rangle$ map based on the four spectral measurements in the energy range 1 to $6 \mathrm{MeV}$ (split into $1-\mathrm{MeV}$ bins). The locations of the four directors are marked with black squares. success of any application of this method depends greatly on where the detectors happen to be placed relative to whatever asymmetries are present. If, as complementary experiments in room-temperature plastic shell implosions suggest, there is a systematic $\lambda=1$ mode, ${ }^{19}$ the locations for the new detectors can be optimally prescribed to detect the mode for these cryogenic implosions.

\section{INFERRING ION-TEMPERATURE}

The width around the primary peak in the neutron spectrum for D-T and D-D fusion neutrons depends on ion temperatures and fluid flow. The variance $\sigma_{\mathrm{n}}{ }^{2}$ in energy for a primary spectrum is given by ${ }^{20}$

$$
\sigma_{\mathrm{n}}^{2}=\frac{2 m_{\mathrm{n}} T_{\mathrm{i}} E_{0}}{m_{\mathrm{n}}+m_{\alpha}}+2 m_{\mathrm{n}} E_{0} \sigma_{v}^{2}
$$

where $m_{\mathrm{n}}$ is the mass of a neutron, $E_{0}$ is the mean energy of the primary neutron $(14.1 \mathrm{MeV}$ for D-T primary neutrons and $2.45 \mathrm{MeV}$ for D-D primary neutrons), $m_{\alpha}$ is the mass of the non-neutron product $\left({ }^{4} \mathrm{He}\right.$ for DT and ${ }^{3} \mathrm{He}$ for $\left.\mathrm{D}_{2}\right)$, and $\sigma_{v}$ is the variance in the component of the fluid velocity along the spectrometer line of sight; i.e.,

$$
\sigma_{v}^{2}=\operatorname{Var}(\vec{v} \cdot \vec{d}) .
$$

The inferred temperature from the neutron spectrum is given by $T_{\text {fit }}$ (Ref. 21)

$$
T_{\text {fit }}=\frac{\Delta E_{\text {fit }}^{2}}{E_{0}} \frac{m_{\mathrm{n}}+m_{\alpha}}{16 m_{\mathrm{n}} \log 2},
$$

where $\Delta E_{\text {fit }}$ is the full width at half maximum (FWHM) of the spectrum. Therefore, the ion temperature can be inferred by measuring the primary neutron spectra. Note that effects such as fluid velocity or background contributions to the neutron spectrum, which widen D-T or D-D primary spectrum, will increase the apparent $\mathrm{D}-\mathrm{T}$ or $\mathrm{D}-\mathrm{D}$ ion temperatures as seen from that direction (Fig. 13 and Table II). The neutron spectra around the D-D and D-T fusion neutron peak [Fig. 13(a)] are broadened because of these effects causing a significant increase in apparent ion temperature (Table II). The apparent temperature calculated from just the D-D primary spectra is $2.97 \mathrm{keV}$ compared to the thermal value of $3 \mathrm{keV}$, while that calculated from the spectra including contributions from background neutrons and excluding fluid flow and without any corrections to the background is $3.89 \mathrm{keV}-\mathrm{a}$ $31 \%$ increase.

These effects are relatively insignificant when inferred from the D-T neutron spectrum [Fig. 13(b)]. This is because (1) the scattering cross sections of $14.1-\mathrm{MeV}$ neutrons are smaller compared to those of $2.45-\mathrm{MeV}$ neutrons and (2) there are no neutrons above $12 \mathrm{MeV}$ from background interactions. As Table II indicates, the increase is less than $1 \%$.

The second term in Eq. (9), corresponding to fluid flow, can significantly affect neutron spectral widths. To isolate the effect of fluid flow, the same hydrocode profile from Fig. 11 was used and only D-T and D-D primary neutrons were tracked. Figure 14 shows that the profile contains a jet of 
(a)

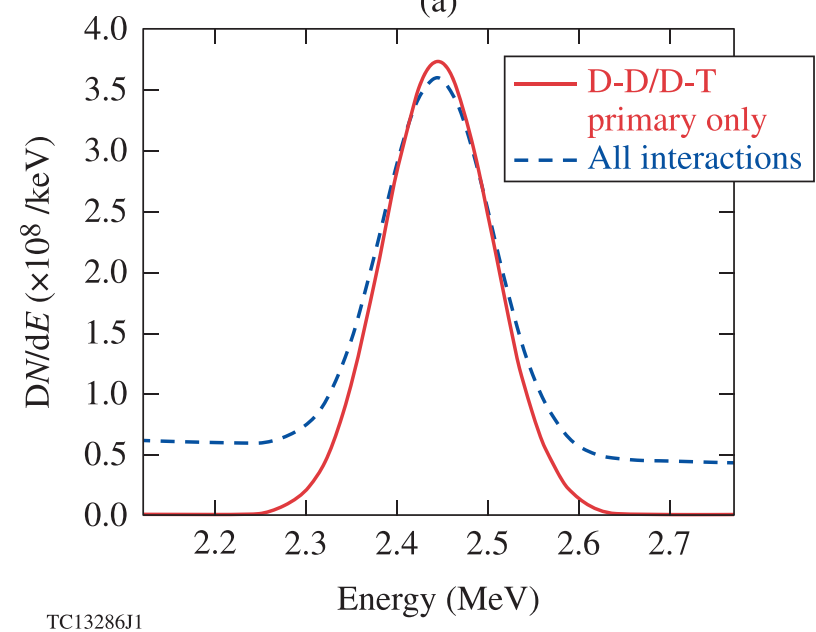

(b)

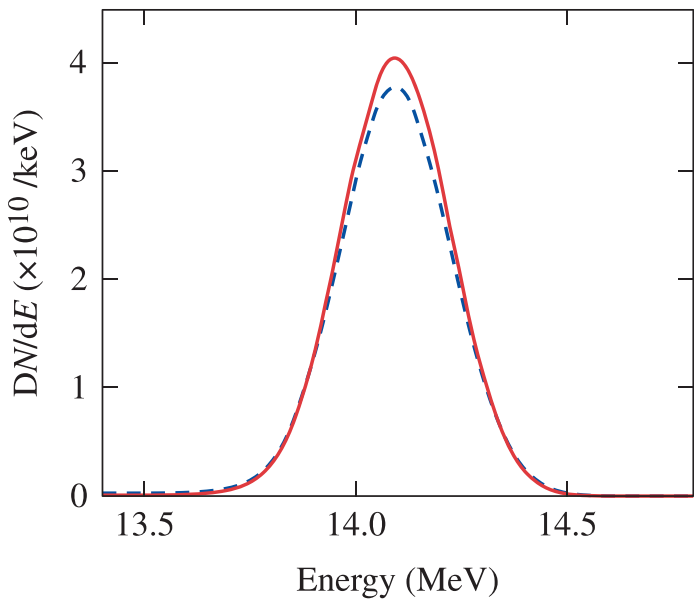

FIG. 13. (a) D-D neutron spectrum, generated by IRIS3D for the profile shown in Fig. 2 with (dashed) and without (solid) neutrons from other interactions included. Note that fluid flow is not accounted for in either case. (b) Same as (a), but for the D-T primary peak. The D-T and D-D temperatures inferred from each spectrum are shown in Table II.

fluid flow directed from the high-density region of the $\lambda=1$ mode to the low-density side. The inferred ion temperatures from D-T and D-D fusion neutron spectra (Fig. 15) correspond closely to this flow. Observed temperatures for both D-T and D-D neutrons are highest at the positions corresponding to the extremes in density shown in Fig. 11(b) and lowest in a ring at about $90^{\circ}$ to these two points. For any direction in the ring around the $\lambda=1$ mode, the jet seen in Fig. 14 points orthogonally to that direction, so it has a very small effect on the value of $\sigma_{v}^{2}$ as seen from that direction as calculated in Eq. (9). Since $\sigma_{v}^{2}$ is not particularly high, the apparent temperature is not particularly higher than what it would be with no fluid flow. For the directions that point along the $\lambda=1$ mode, however, there is a large variation in $\vec{v} \cdot \vec{d}$ [Eq. (9)] leading to a large $\sigma_{\mathrm{n}}^{2}$ [Eq. (8)]. As a result, the apparent temperature is much higher when considering fluid flow in these directions. As Fig. 15 indicates, the temperature along the direction of the jet inferred from DT neutrons has a value of $4.4 \mathrm{keV}$, whereas the value inferred from DD neutrons has a value of $3.9 \mathrm{keV}$. Both these values are higher than the spatially weighted thermal temperature of $3.1 \mathrm{keV}$. These results indicate that with multiple views of the primary neutron spectra, the relative ion temperature values inferred can provide an indication of low-order hot-spot asymmetries. Note that the variation in ion temperature follows the inferred areal density map shown in Fig. 12 with some differences. For this dominant $\lambda=1$ mode, the inferred areal density has a maximum in one direction along the jet and a minimum in the other direction. The ion temperature is

TABLE II. D-D and D-T apparent ion temperatures inferred from the neutron spectra shown in Fig. 13. These values should be compared to a thermal temperature of $3 \mathrm{keV}$.

\begin{tabular}{lcc}
\hline \hline Inferred ion temperature $(\mathrm{keV})$ & D-D reaction & D-T reaction \\
\hline Only primary neutrons & 3.0 & 2.9 \\
All neutron interactions & 3.9 & 3.0 \\
\hline \hline
\end{tabular}

symmetric along the jet axis. This complicates the correlation of areal density asymmetries with ion temperature variations. Multi-mode simulations will be post-processed to pin down any possible correlation between shell density asymmetries and fluid flow in the hot spot by comparing the inferred ion temperatures and areal densities.

\section{CONCLUSIONS}

Long-wavelength asymmetries have been shown to influence performance in OMEGA-scale inertial confinement fusion implosions. Using a newly developed particle tracking code (IRIS3D, see the Appendix), neutron-based spectra are used to study signatures of asymmetry in OMEGA-scale cryogenic implosions. These include areal-density $(\langle\rho L\rangle)$ variations and neutron spectral-width variations around the compressed target. Subtraction of neutrons from the deuteron break-up reaction and $\mathrm{T}-\mathrm{T}$ fusion is extremely important for the lower-energy range ( 1 to $6 \mathrm{MeV})$ in the spectrum to infer true areal densities. It is shown that even with a single view, a $\langle\rho L\rangle$ map of the implosion can be obtained by mapping

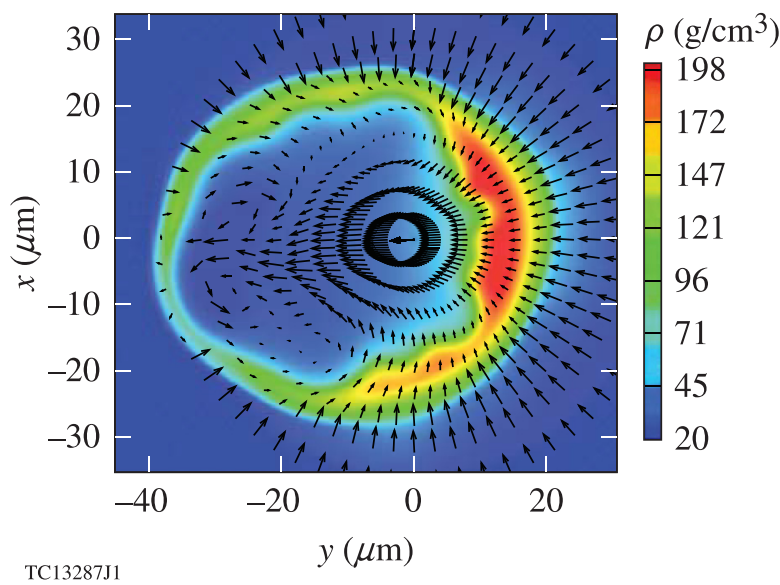

FIG. 14. Same as Fig. 11(a), but with additional vectors representing fluid velocity direction and magnitude. 
(a)

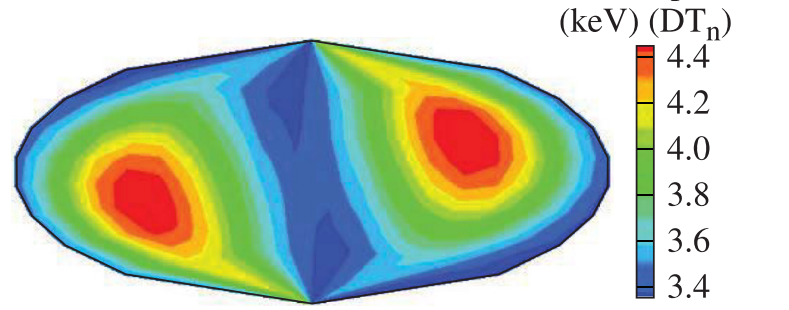

(b)

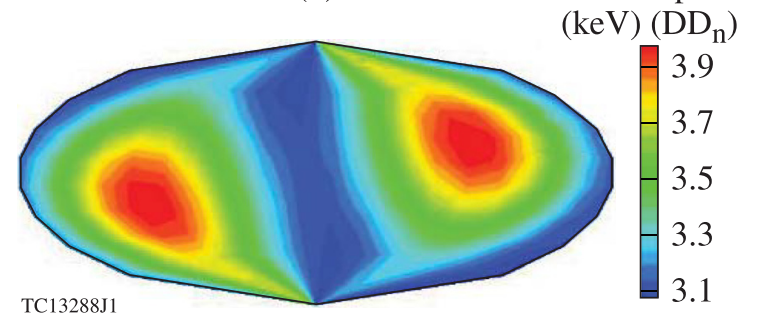

FIG. 15. (a) Hammer plot of apparent DT ion temperatures at the time of peak neutron production in Fig. 11, as calculated by IRIS3D considering DT neutrons only. (b) Same as (a), but for apparent DD ion temperatures.

different energies in the neutron spectrum to different parts of the implosion using kinematic relations of the elastic scattering reactions. Multiple views can improve the areal density coverage of the implosion and provide information on the underlying asymmetric structure of the compressed shell. An additional neutron time-of-flight detector is being implemented along a third line of sight. Background subtraction from the lower energy D-D fusion peak is critical for reliably inferring the width of the spectrum. It is also shown that measurements of neutron width can be correlated with an overall direction of fluid flow from both $\mathrm{D}-\mathrm{D}$ and $\mathrm{D}-\mathrm{T}$ ion temperatures, provided the background correction has been performed for the D-D temperature. Detailed simulations and comparisons with experiment for the range of implosion parameters will be presented elsewhere. We will continue to use IRIS3D as a postprocessor to 3-D hydrodynamic codes and will pursue detailed comparisons with observations for OMEGA cryogenic implosions.

\section{ACKNOWLEDGMENTS}

The authors thank Dr. Ken Anderson for providing the profiles from HYDRA simulations used in this paper. This material is based upon work supported by the Department of Energy National Nuclear Security Administration under Award No. DE-NA0001944, the University of Rochester, and the New York State Energy Research and Development Authority.

This report was prepared as an account of work sponsored by an agency of the U.S. Government. Neither the U.S. Government nor any agency thereof, nor any of their employees, makes any warranty, express or implied, or assumes any legal liability or responsibility for the accuracy, completeness, or usefulness of any information, apparatus, product, or process disclosed, or represents that its use would not infringe privately owned rights. Reference herein to any specific commercial product, process, or service by trade name, trademark, manufacturer, or otherwise does not necessarily constitute or imply its endorsement, recommendation, or favoring by the U.S. Government or any agency thereof. The views and opinions of authors expressed herein do not necessarily state or reflect those of the U.S. Government or any agency thereof.

\section{APPENDIX: STRUCTURE AND METHODS OF IRIS3D}

IRIS 3D is a parallel Monte Carlo-based neutron-tracking code. Neutrons generated by a variety of interactions are tracked through a spherical grid made up of hexahedral cells (Fig. 16). Note that in Fig. 16 and throughout this Appendix, $r, \theta$, and $\varphi$ refer to spherical coordinates with $\theta$ denoting the polar angle and $\varphi$ denoting the azimuthal angle. As described in the figure, each cell is indexed and is characterized by a DT fuel density, an ion temperature, and a fluid vector velocity. While the grid mostly comprises these cells, it also contains a few special zones. To preserve the hexahedral structure of each cell, IRIS3D imposes exclusion zones within a small distance of the origin and within a small angle of each pole that are not occupied by cells. Therefore, each vertex with an $i$-index of zero is not at $r=0$ but at a very small distance from the origin, and each vertex with a $j$-index of zero or the maximum index is not at $\theta=0$ or $\pi$, respectively, but at 0 plus a small angle and $\pi$ minus a small angle, respectively. These exclusion zones are small enough that a very limited number of particles can pass through them in a given simulation. They have a minimal effect on results, and particles that do pass through them are simply propagated across them by a reflection, where they continue on through the remainder of the grid as usual.

IRIS3D currently models six neutron-emitting interactions. Spectra from these interactions are shown in Fig. 6: primary neutron-producing fusion reactions are $d(t, n)^{4} \mathrm{He}$, $\mathrm{d}(\mathrm{d}, \mathrm{n})^{3} \mathrm{He}$, and $\mathrm{t}(\mathrm{t}, 2 \mathrm{n})^{4} \mathrm{He}$; and three secondary interactions are $d\left(n, n^{\prime}\right) d^{\prime}, t\left(n, n^{\prime}\right) t^{\prime}$, and $d(n, 2 n) p$. The secondary interactions involve the interactions of primary neutrons as they traverse the grid.

A trajectory in IRIS3D is generated in some cell, $(i, j, k)$, representing some number of neutrons $y_{\mathrm{n}}$, at some energy $E$, which move in some direction $\vec{d}=(u, v, w)$, where $\vec{d}$ is a unit vector and $u, v$, and $w$ are the direction cosines. The

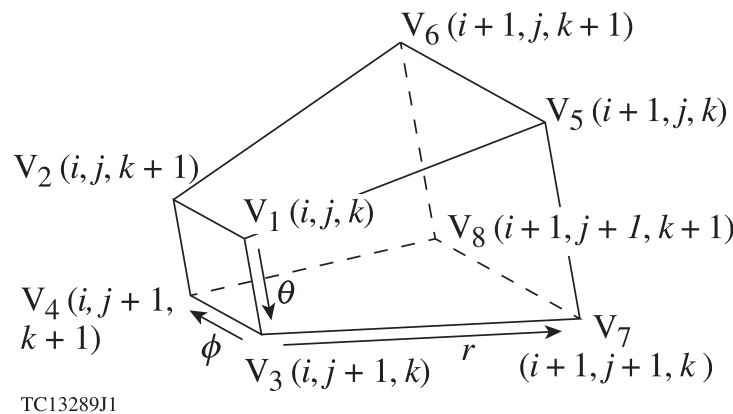

FIG. 16. Layout of a generic hexahedral cell in IRIS3D. Each vertex is represented by indices $i, j$, and $k$, which increase with increasing $r, \theta$, and $\varphi$, respectively, and each cell is bounded by eight vertices as shown. 
initial position of the trajectory, $P_{0}=(x, y, z)$, is set as the centroid of the cell in which it is generated. The trajectory is tracked in a straight line through the grid.

For each cell the trajectory enters, the following process is carried out: First, initial points $Q$ and normal vectors for the six planes of the cell are calculated. Next, for each plane, the following calculation is performed:

$$
\Delta s=\frac{\overrightarrow{P Q} \cdot \vec{n}}{\vec{d} \cdot \vec{n}},
$$

where $P$ denotes the initial position of the trajectory within the cell and $\Delta s$ represents the (possibly negative) distance the trajectory must travel along its direction to intersect with that plane. The plane that yields the smallest positive value of $\Delta s$ is the next plane with which the trajectory will intersect, so it corresponds to the face of the cell through which the trajectory will exit. Next, any secondary neutron interactions the user has specified to be considered are calculated based on the physical parameters of the cell and the neutrons represented by the trajectory along with the value of $\Delta s$. Finally, the position $P$ of the particle is changed to the position of intersection with that face and then propagated a small distance along direction $d$, and the particle's current cell index $(i, j, k)$ is updated based on the face through which it exited. For example, if the particle started in cell $(i, j, k)$ and exited through the face lying in the plane $V_{1} V_{2} V_{3}$, its new cell index would be $(i-1, j, k)$, as is apparent from Fig. 16. If, at this point, the cell indices indicate that the trajectory has entered an exclusion zone, as described above, the particle is appropriately reflected to continue along the grid. For each trajectory, this process is carried out until the particle exits the grid. When this occurs, the trajectory is binned. Since the imploded core is very small compared to the target chamber, the target can be treated as a source when viewed from any spectrometer, so trajectories are binned only according to their direction $\vec{d}$.

IRIS3D accounts for two different types of detectors: First, a grid of detectors is placed around the target. The grid lines are at constant $\theta$ or $\varphi$ values, so for each trajectory, its $(\theta, \varphi)$ direction is calculated according to $d$, and it is binned in whichever grid rectangle its $(\theta, \varphi)$ direction lies. In addition, detectors at specific $(\theta, \varphi)$ locations and with specific solid angles $\Omega$ are specified by the user (for example, these would correspond to existing detector locations on OMEGA).

Neutron trajectories are binned into spectra at a given spectrometer if and only if

$$
\vec{d} \cdot \vec{l} \geq 1-\frac{\Omega}{2 \pi},
$$

where $\vec{l}$ is a unit vector in the direction of the detector. In the calculation, spectrometers record both time-integrated and time-resolved neutron spectra.

In what follows, we discuss in detail how trajectories are launched. First, the number of primary fusion reactions in each cell is calculated. To do this, a reactivity $\langle\sigma v\rangle$ is calculated in each cell based on cell ion temperatures using the analytic fit given in Ref. 22. The number of fusion reactions $y_{\text {cell }}$ is calculated as

$$
y_{\text {cell }}=n_{\mathrm{D}} n_{\mathrm{T}}\langle\sigma v\rangle V \Delta t=f_{\mathrm{D}} f_{\mathrm{T}}\left(\frac{\rho}{m}\right)^{2}\langle\sigma v\rangle V \Delta t,
$$

where $n_{\mathrm{i}}$ and $n_{\mathrm{j}}$ are the ion densities for the two different ions; $f_{i}$ and $f_{j}$ are the ion fractions in the fuel characterized during the target fabrication process; $V$ is the cell volume; $\Delta t$ is the duration of the time slice being processed for neutron spectra; and $\delta_{i j}$ is the Kronecker delta $(=1$ if $i=j$, i.e., or D-D fusion and 0 otherwise). If $N$ is the total number of trajectories that are to be launched across the entire grid for that time step (determined by requiring adequate statistics in the calculated spectra), then the number of trajectories launched per cell is $y_{\text {cell }} / \sum y_{\text {cell }} \cdot N$.

For each primary neutron trajectory launched from a cell centroid, $d$ is chosen as a random unit vector (since the emission of primary neutrons is isotropic) and $E$ sampled from a normal distribution with mean $\mu$ and standard deviation $\sigma$ determined by ${ }^{20}$

$$
\mu=E_{0}+(\vec{v} \cdot \vec{d}) \sqrt{2 m_{\mathrm{n}} E_{0}}
$$

and

$$
\sigma=\sqrt{\frac{2 m_{\mathrm{n}} T_{\mathrm{i}} E_{0}}{m_{\mathrm{n}}+m^{4} \mathrm{He}}} .
$$

Here $E_{0}=14.1 \mathrm{MeV}$ is the mean neutron energy from the D-T fusion reaction; $\vec{v}$ the cell fluid velocity; $m_{\mathrm{n}}$ and $m^{4} \mathrm{He}$ are the masses of the neutron and alpha particles, respectively; and $T_{\mathrm{i}}$ is the cell ion temperature. The second term in the expression for the average energy takes into account the shift in the energy of the neutron caused by the fluid velocity. The expression for the standard deviation takes into account the broadening of the neutron spectrum caused by the plasma temperature. $^{21}$

$\mathrm{T}-\mathrm{T}$ reactions are treated similarly. Reactivity for temperatures below $1 \mathrm{keV}$ is calculated using the analytic fit given in Ref. 23, while reactivity for ion temperatures above or equal to $1 \mathrm{keV}$ is calculated by linear interpolation using the lookup table given in Ref. 24. The initial neutron energy $E_{0}$ is sampled from a distribution obtained by an $R$-matrix calculation, ${ }^{25}$ which agrees well with experimental measurements. ${ }^{25}$ Note that the primary neutrons from the T-T reaction are not launched in pairs that obey the conservation laws but instead are launched one at a time, independently. Momentum and energy are conserved only with adequate statistics.

Secondary reactions are considered next. IRIS3D starts by estimating the total number of deuteron-scattered neutrons

$$
y^{\prime}=y_{\mathrm{n}} \frac{f_{\mathrm{D}} \sigma_{14.1}}{\bar{m}} \rho R,
$$

where $y_{\mathrm{n}}$ is the total number of primary neutrons generated $\left(=\sum y_{\text {cell }}\right), \sigma_{14.1}$ is the cross section for the $14.1-\mathrm{MeV}$ neutrons elastically scattering off the deuteron ions, and $\rho R$ is a spatially averaged areal density. The scattering cross section is obtained from an energy-dependent look-up table given by Ref. 26. The estimate is used to launch trajectories 
representing the secondary particles from the cell. Note that any inaccuracy in this estimated value of secondary particles causes only the number of secondary trajectories launched to differ from the desired number of trajectories and does not affect the accuracy of the results. The number $N$ of secondary trajectories launched from a given interaction location is a code input to obtain converged results; therefore, the weight of each trajectory is $y_{0}=y^{\prime} / N$.

Each trajectory is subdivided into discrete path lengths $\Delta s$ and neutrons are transported in units of this path length. The number of deuteron (or triton)-scattering interactions, $y_{\mathrm{n}}^{\prime}$, is calculated as

$$
y^{\prime}=y \frac{f_{\mathrm{D} / \mathrm{T}} \sigma_{\mathrm{D} / \mathrm{T}}}{\bar{m}} \rho \Delta s,
$$

where $y$ is the number of neutrons represented by the trajectory and $\sigma$ is the cross section for $14.1-\mathrm{MeV}$ neutrons elastically scattering off deuterons or tritons with energy $E$. At this point, $y$ is replaced with $y-y^{\prime}$ since the primary trajectory loses any neutrons that scatter away. The weight of the trajectory is $y_{0}$, and a scattering angle $\theta$ is determined by interpolating between a set of energy-dependent angular cross sections from Ref. 16. An azimuthal scattering angle $\varphi$ is then picked randomly over the interval $(0,2 \pi)$, and the scattered neutron trajectory direction $\vec{d}^{\prime}$ is defined by

$$
\vec{d}^{\prime}=\vec{a} \sin \theta \cos \phi+\vec{b} \sin \theta \sin \phi+\vec{d} \cos \theta,
$$

where $\vec{d}$ is the direction of the original trajectory and $\vec{a}$ and $\vec{b}$ are a set of unit vectors that are orthogonal to each other and to $\vec{d}$. Finally, $E^{\prime}$, the energy of the scattered neutron, is determined based on $\theta$ using Eq. (7). Once generated, the scattered trajectory is again handled as described previously.

The deuteron-breakup reaction is modeled somewhat differently. A look-up table for angular distributions of emerging particles is available only for $14.1-\mathrm{MeV}$ incident neutrons, so the interaction is considered only in the case where the original trajectory represents $\mathrm{D}-\mathrm{T}$ primary neutrons. A constant value of $\sigma=\sigma_{14.1}=164.821 \mathrm{mb}$ is used for both Eqs. (A6) and (A7) (see Ref. 26). Two neutron trajectories are launched for each breakup reaction. Since there is no deterministic relationship between the angle and energy of each emitted neutron, the angle of the emitted neutron is sampled from a distribution calculated based on Ref. 27 and energy is sampled from a distribution based on that scattering angle. As was the case with the T-T primary reaction, the neutrons' energies and scattering angles are both sampled independently, so momentum and energy are not necessarily conserved in any particular interaction, but conservation improves with increasingly better statistics.

If a series of time slices from the same implosion are used instead, the process is repeated for each time step and the results are accumulated over time.

${ }^{1}$ J. Nuckolls, L. Wood, A. Thiessen, and G. Zimmerman, Nature 239, 139 (1972).

${ }^{2}$ T. R. Boehly, D. L. Brown, R. S. Craxton, R. L. Keck, J. P. Knauer, J. H. Kelly, T. J. Kessler, S. A. Kumpan, S. J. Loucks, S. A. Letzring, F. J.
Marshall, R. L. McCrory, S. F. B. Morse, W. Seka, J. M. Soures, and C. P. Verdon, Opt. Commun. 133, 495 (1997).

${ }^{3}$ J. Delettrez, R. Epstein, M. C. Richardson, P. A. Jaanimagi, and B. L. Henke, Phys. Rev. A 36, 3926 (1987).

${ }^{4}$ M. M. Marinak, G. D. Kerbel, N. A. Gentile, O. Jones, D. Munro, S. Pollaine, T. R. Dittrich, and S. W. Haan, Phys. Plasmas 8, 2275 (2001).

${ }^{5}$ L. Rayleigh, Proc. London Math Soc. XIV, 170 (1883); R. Kishony and D. Shvarts, Phys. Plasmas 8, 4925 (2001).

${ }^{6}$ C. Stoeckl et al., Phys. Plasmas 24, 056304 (2017).

${ }^{7}$ F. J. Marshall, R. E. Bahr, V. N. Goncharov, V. Y. Glebov, B. Peng, S. P. Regan, T. C. Sangster, and C. Stoeckl, Rev. Sci. Instrum. 88, 093702 (2017).

${ }^{8}$ R. Betti, P. Y. Chang, B. K. Spears, K. S. Anderson, J. Edwards, M. Fatenejad, J. D. Lindl, R. L. McCrory, R. Nora, and D. Shvarts, Phys. Plasmas 17, 058102 (2010).

${ }^{9}$ D. C. Wilson, W. C. Mead, L. Disdier, M. Houry, J. L. Bourgade, and T. J. Murphy, Nucl. Instrum. Methods Phys. Res., Sect. A 488, 400 (2002).

${ }^{10}$ J. A. Frenje, D. T. Casey, C. K. Li, J. R. Rygg, F. H. Séguin, R. D. Petrasso, V. Y. Glebov, D. D. Meyerhofer, T. C. Sangster, S. Hatchett, S. Haan, C. Cerjan, O. Landen, M. Moran, P. Song, D. C. Wilson, and R. J. Leeper, Rev. Sci. Instrum. 79, 10E502 (2008).

${ }^{11}$ C. J. Forrest, P. B. Radha, V. Y. Glebov, V. N. Goncharov, J. P. Knauer, A. Pruyne, M. Romanofsky, T. C. Sangster, M. J. Shoup III, C. Stoeckl, D. T. Casey, M. Gatu-Johnson, and S. Gardner, Rev. Sci. Instrum. 83, 10D919 (2012).

${ }^{12}$ T. J. Murphy, Phys. Plasmas 21, 072701 (2014).

${ }^{13}$ ENDF/B-N1.1, IAEA Nuclear Data Services.

${ }^{14}$ S. Skupsky and S. Kacenjar, J. Appl. Phys. 52, 2608 (1981).

${ }^{15}$ B. Appelbe and J. Chittenden, Plasma Phys. Controlled Fusion 53, 045002 (2011).

${ }^{16}$ J. A. Frenje, C. K. Li, F. H. Séguin, D. T. Casey, R. D. Petrasso, D. P. McNabb, P. Navratil, S. Quaglioni, T. C. Sangster, V. Y. Glebov, and D. D. Meyerhofer, Phys. Rev. Lett. 107, 122502 (2011)

${ }^{17}$ C. K. Li, F. H. Séguin, D. G. Hicks, J. A. Frenje, K. M. Green, S. Kurebayashi, R. D. Petrasso, D. D. Meyerhofer, J. M. Soures, V. Y. Glebov, R. L. Keck, P. B. Radha, S. Roberts, W. Seka, S. Skupsky, C. Stoeckl, and T. C. Sangster, Phys. Plasmas 8, 4902 (2001).

${ }^{18}$ D. L. Bleuel, C. B. Yeamans, L. A. Bernstein, R. M. Bionta, J. A. Caggiano, D. T. Casey, G. W. Cooper, O. B. Drury, J. A. Frenje, C. A. Hagmann, R. Hatarik, J. P. Knauer, M. G. Johnson, K. M. Knittel, R. J. Leeper, J. M. McNaney, M. Moran, C. L. Ruiz, and D. H. G. Schneider, Rev. Sci. Instrum. 83, 10D313 (2012).

${ }^{19}$ R. C. Shah, B. M. Haines, F. J. Wysocki, J. F. Benage, J. A. Fooks, V. Glebov, P. Hakel, M. Hoppe, I. V. Igumenshchev, G. Kagan, R. C. Mancini, F. J. Marshall, D. T. Michel, T. J. Murphy, M. E. Schoff, K. Silverstein, C. Stoeckl, and B. Yaakobi, Phys. Rev. Lett. 118, 135001 (2017).

${ }^{20}$ V. N. Goncharov, T. C. Sangster, R. Betti, T. R. Boehly, M. J. Bonino, T. J. B. Collins, R. S. Craxton, J. A. Delettrez, D. H. Edgell, R. Epstein, R. K. Follet, C. J. Forrest, D. H. Froula, V. Yu. Glebov, D. R. Harding, R. J. Henchen, S. X. Hu, I. V. Igumenshchev, R. Janezic, J. H. Kelly, T. J. Kessler, T. Z. Kosc, S. J. Loucks, J. A. Marozas, F. J. Marshall, A. V. Maximov, R. L. McCrory, P. W. McKenty, D. D. Meyerhofer, D. T. Michel, J. F. Myatt, R. Nora, P. B. Radha, S. P. Regan, W. Seka, W. T. Shmayda, R. W. Short, A. Shvydky, S. Skupsky, C. Stoeckl, B. Yaakobi, J. A. Frenje, M. Gatu-Johnson, R. D. Petrasso, and D. T. Casey, Phys. Plasmas 21, 056315 (2014).

${ }^{21}$ H. Brysk, Plasma Phys. 15, 611 (1973).

${ }^{22}$ H. S. Bosch and G. M. Hale, Nucl. Fusion 32, 611 (1992).

${ }^{23}$ A. Peres, J. Appl. Phys. 50, 5569 (1979).

${ }^{24}$ J. R. McNally, Jr., K. E. Rothe, and R. D. Sharp, Oak Ridge National Laboratory Report ORNL/TM-6914, 1979.

${ }^{25}$ D. B. Sayre, C. R. Brune, J. A. Caggiano, V. Y. Glebov, R. Hatarik, A. D. Bacher, D. L. Bleuel, D. T. Casey, C. J. Cerjan, M. J. Eckart, R. J. Fortner, J. A. Frenje, S. Friedrich, M. Gatu-Johnson, G. P. Grim, C. Hagmann, J. P. Knauer, J. L. Kline, D. P. McNabb, J. M. McNaney, J. M. Mintz, M. J. Moran, A. Nikroo, T. Phillips, J. E. Pino, B. A. Remington, D. P. Rowley, D. H. Schneider, V. A. Smalyuk, W. Stoeffl, R. E. Tipton, S. V. Weber, and C. B. Yeamans, Phys. Rev. Lett. 111, 052501 (2013).

${ }^{26}$ P. G. Young, G. M. Hale, and M. G. Chadwick, ENDF/B-VII.1, IAEA Nuclear Data Services, 22 December 2011.

${ }^{27}$ A. Deltuva, private communication, Institute of Theoretical Physics and Astronomy, Vilinus University, Vilinus, Lithuania (2016). 